Corresponding authors: Deyle. David@mayo.edu; Klee.Eric@ mayo.edu

(c) 2017 Cousin et al. This article is distributed under the terms of the Creative Commons

Attribution-NonCommercial License, which permits reuse and redistribution, except for commercial purposes, provided that the original author and source are credited.

Ontology terms: aneurysm of an abdominal artery; aortic root dilatation; arterial tortuosity; dental crowding; dolichocephaly; hip subluxation; inguinal hernia; joint laxity; malar flattening; pes planus; shoulder subluxation; arthralgia/arthritis

Published by Cold Spring Harbor Laboratory Press

doi: $10.1101 /$ mcs.a001727

\section{Functional validation reveals the novel missense V419L variant in TGFBR2 associated with Loeys-Dietz syndrome (LDS) impairs canonical TGF- $\beta$ signaling}

\author{
Margot A. Cousin, ${ }^{1,2}$ Michael T. Zimmermann, ${ }^{1}$ Angela J. Mathison, ${ }^{3}$ \\ Patrick R. Blackburn, ${ }^{4,5}$ Nicole J. Boczek, ${ }^{1,2}$ Gavin R. Oliver, ${ }^{1}$ Gwen A. Lomberk, ${ }^{3,6}$ \\ Raul A. Urrutia, ${ }^{2,3}$ David R. Deyle, ${ }^{2,7}$ and Eric W. Klee $e^{1,2,7}$

\begin{abstract}
${ }^{1}$ Department of Health Sciences Research, Mayo Clinic, Rochester, Minnesota 55905, USA; ${ }^{2}$ Center for Individualized Medicine, Mayo Clinic, Rochester, Minnesota 55905, USA; ${ }^{3}$ Laboratory of Epigenetics and Chromatin Dynamics, Gastroenterology Research Unit, Department of Medicine, Mayo Clinic, Rochester, USA; ${ }^{5}$ Center for Individualized Medicine, Mayo Clinic, Jacksonville, Florida 32224, USA; ${ }^{6}$ Department of Molecular Pharmacology and Experimental Therapeutics, Mayo Clinic, Rochester, Minnesota 55905, USA; ${ }^{7}$ Department of Clinic Genomics, Mayo Clinic, Rochester, Minnesota 55905, USA
\end{abstract} \\ Minnesota 55905, USA; ${ }^{4}$ Department of Health Sciences Research, Mayo Clinic, Jacksonville, Florida 32224,
}

\begin{abstract}
TGF- $\beta$-related heritable connective tissue disorders are characterized by a similar pattern of cardiovascular defects, including aortic root dilatation, mitral valve prolapse, vascular aneurysms, and vascular dissections and exhibit incomplete penetrance and variable expressivity. Because of the phenotypic overlap of these disorders, panel-based genetic testing is frequently used to confirm the clinical findings. Unfortunately in many cases, variants of uncertain significance (VUSs) obscure the genetic diagnosis until more information becomes available. Here, we describe and characterize the functional impact of a novel VUS in the TGFBR2 kinase domain (c.1255G>T; p.Val419Leu), in a patient with the clinical diagnosis of Marfan syndrome spectrum. We assessed the structural and functional consequence of this VUS using molecular modeling, molecular dynamic simulations, and in vitro cell-based assays. A high-quality homology-based model of TGFBR2 was generated and computational mutagenesis followed by refinement and molecular dynamics simulations were used to assess structural and dynamic changes. Relative to wild type, the V419L induced conformational and dynamic changes that may affect ATP binding, increasing the likelihood of adopting an inactive state, and, we hypothesize, alter canonical signaling. Experimentally, we tested this by measuring the canonical TGF- $\beta$ signaling pathway activation at two points; V419L significantly delayed SMAD2 phosphorylation by western blot and significantly decreased TGF- $\beta$-induced gene transcription by reporter assays consistent with known pathogenic variants in this gene. Thus, our results establish that the V419L variant leads to aberrant TGF- $\beta$ signaling and confirm the diagnosis of Loeys-Dietz syndrome in this patient.
\end{abstract}

[Supplemental material is available for this article.]

\section{INTRODUCTION}

Rare diseases are individually rare but collectively common, affecting approximately 25-30 million individuals in the United States today, and $>80 \%$ of rare diseases have a genetic etiology (Global Genes 2016). Translation of the recent technological advances in genomic 
testing into clinical practice has enabled an increase in the availability and use of clinical genetic testing to determine the etiology of a patient's suspected Mendelian disease. Establishing a genetic diagnosis can ensure appropriate medical management of patients but can also inform family members who may be at risk of harboring the same variant and for family planning. Our ability to interpret the genetic variation we uncover with these technologies, however, is lagging behind our ability to confidently identify them. As a consequence, a majority of the genetic variation uncovered are variants of uncertain significance (VUSs) and additional efforts are needed to determine whether these VUSs are benign or pathogenic. Factors contributing to the challenges of VUS interpretation include lack of coobservation of individual VUS in similarly affected patients, lack of related phenotypic evidence for the harboring gene, and the precise mechanism of disease may be unclear without gold-standard clinical tests to verify the link between a gene's dysfunction and a clinical diagnosis.

Several heritable connective tissue disorders are associated with dysfunction in the TGF- $\beta$ signaling pathway (Fig. 1) and have specific challenges for determining pathogenicity of variants in the associated genes. The known disease-causal genes in the TGF- $\beta$ pathway and associated disorders include FBN1 (Marfan syndrome), TGFBR1, TGFBR2, SMAD3, TGFB2, and TGFB3 (Loeys-Dietz syndrome [LDS]), SMAD2 (SMAD2-related arterial aneurysm disorder), and SKI (Shprintzen-Goldberg syndrome). This set of TGF- $\beta$-related connective tissue disorders are autosomal dominantly inherited, have significant phenotypic overlap, and have been well reviewed recently (Verstraeten et al. 2016). These disorders have multisystem involvement with their most significant morbidity and mortality stemming from cardiovascular complications such as arterial dissections, aneurysms, and valve prolapse.

The severe potential outcomes of the arterial dissections and aneurysms associated with the TGF- $\beta$ dysfunction syndromes necessitate determining the genetic etiology to ensure the proper screening of family members and managing risk of complications (De Backer et al. 2013). The interpretation of VUSs in the TGF- $\beta$-related heritable connective tissue disorders, however, has specific challenges. Most variants described have only been observed within a single family and are associated with incomplete penetrance, significant phenotypic variability, and variable expressivity. Thus, discerning variant and disease cosegregation within a family and identifying additional unrelated patients with the same variant may be difficult, limiting the evidence available to define variant pathogenicity according to the current guidelines for variant interpretation (Richards et al. 2015). Consequently, additional efforts are needed to determine whether a VUS may be contributing to disease.

In this study, we used standard variant annotation, paralog analyses, in silico protein modeling, and heterologous cell-based assays to determine the pathogenicity of a clinically

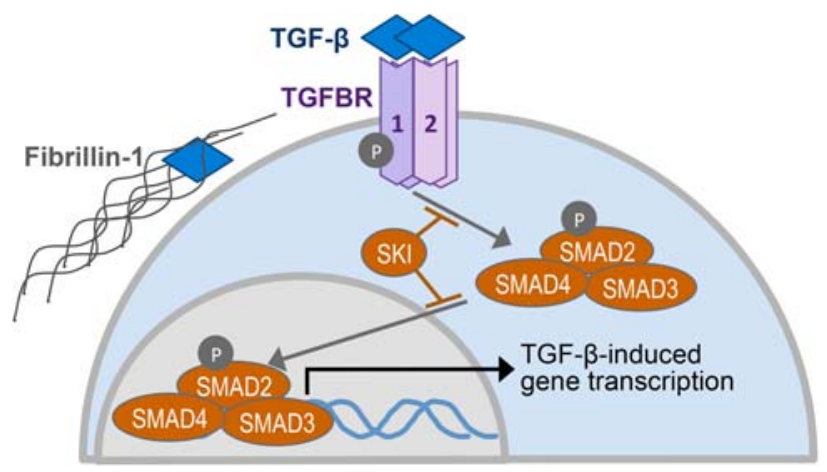

Figure 1. Canonical TGF- $\beta$ signaling pathway. 
detected TGFBR2 VUS in a patient with aortic root dilatation suspected of having an underlying connective tissue disorder. We show how the use of both in silico and in vitro functional assessment can determine the pathogenicity of variants in TGFBR2 and facilitate a genetic diagnosis of Loeys-Dietz syndrome.

\section{RESULTS}

\section{Clinical Findings and Genetic Testing}

The novel missense V419L variant in the TGFBR2 gene characterized in this study was identified by genetic testing of a male patient who presented at the age of $42 \mathrm{yr}$ (Fig. 2A, III-1) to the Mayo Clinic for suspected Marfan syndrome. His previous history included right inguinal hernia repair at $3 \mathrm{yr}$ of age, multiple orthopedic interventions including right anterior cruciate ligament $(\mathrm{ACL})$ reconstruction, arthroscopy of the right hip, and subluxations of his hip, right thumb, and shoulders. He had a history of braces on his teeth and dental overcrowding, a pneumothorax during a bout of bronchitis at $31 \mathrm{yr}$ old, joint laxity, and a 10-yr history of intermittent joint pains that had worsened over the $2 \mathrm{yr}$ prior to presentation.

The family history was significant for a maternal grandfather (Fig. 2A, I-1) who passed away at the age of $54 \mathrm{yr}$ from an unexplained cardiovascular event. Both the patient's mother (II-1) and sister (III-2) were evaluated by echocardiograms and found to have normal aortic roots. The remainder of the family history was unremarkable.

On physical exam the patient was tall with a height of $197.7 \mathrm{~cm}$ (100th percentile; +2.72 $\mathrm{SD}$ ), had dolichocephaly, malar hypoplasia, mild retrognathia, pes planus with hindfoot deformity, and positive wrist and thumb sign. Based on the revised Ghent nosology (Loeys et al. 2010), a score, termed the systemic score, is calculated for patients suspected as having Marfan syndrome whereby the presence of specific phenotypes in the patient are assigned weighted values. With his history of pneumothorax, the systemic score of this patient was 9, and he met the clinical criteria for Marfan syndrome (systemic score $\geq 7$ ). Echocardiogram evaluation identified a dilated sinus of Valsalva of $54 \mathrm{~mm}$ with Z-score of 7.22 (positive systemic score criteria is $\geq 2$ ) shown in Figure $2 \mathrm{~B}$ and additional imaging studies revealed vertebral and cervical internal carotid artery tortuosity. The patient subsequently underwent aortic valve-sparing root replacement with reimplantation of coronary arteries and aortic valve repair at age 42, and aortobiiliac reconstruction at the age of $43 \mathrm{yr}$ for iliac aneurysms (Fig. 2C).

Gene-panel testing for Marfan syndrome and related disorders was pursued through GeneDx and identified missense VUSs (Table 1) in TGFBR2 (NM_003242.5: c.1255G>T; p. V419L), MYH11 (NM_002474.2: c.5585G>A; p.R1862H), and FBN1 (NM_002474.2:

A
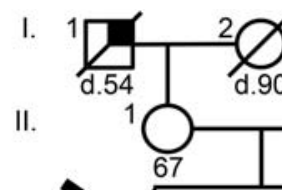

III.

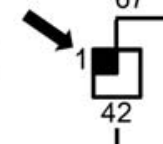

IV.
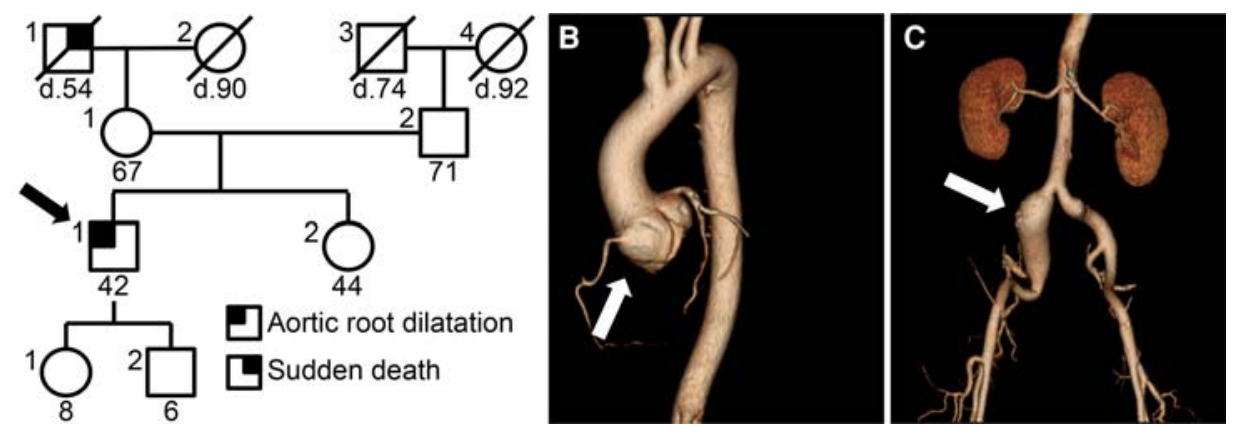

Figure 2. Family history and vascular imaging. (A) Pedigree (arrow indicates proband). (B) Aortic root dilatation and $(C)$ aneurysms of the common iliac arteries of the proband visualized using computer tomography angiography. 


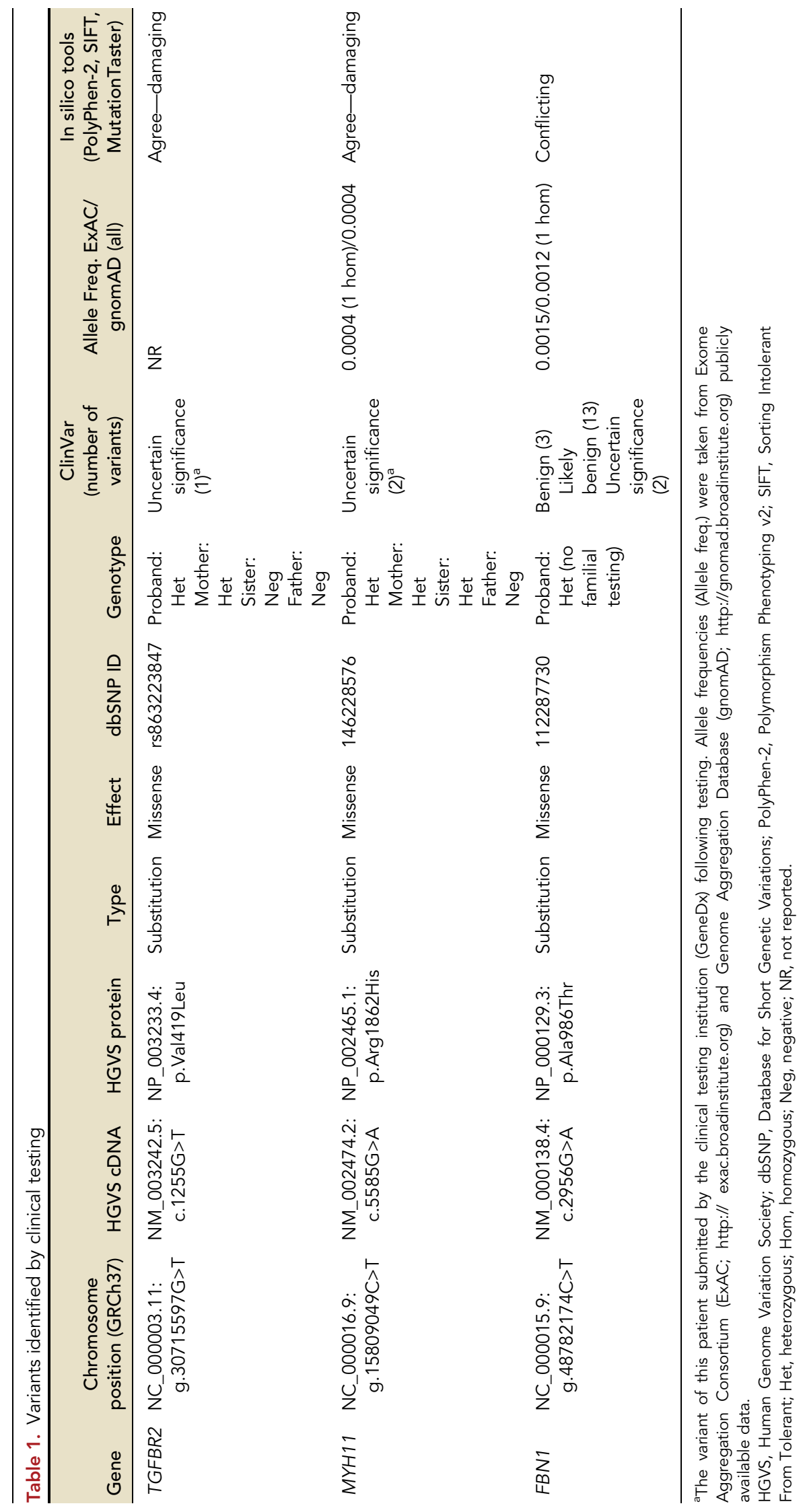


c.2956G >A; p.A986T). Familial testing revealed the patient's mother was heterozygous for the TGFBR2 and MYH11 variants, the sister was heterozygous for the MYH11 variant, and the father was negative for both. Pathogenic variants in TGFBR2 lead to Loeys-Dietz syndrome (MIM \#610168) that closely matches the phenotypic presentation of this patient. Pathogenic variants in FBN1 lead to Marfan syndrome (MIM \#154700); however, the variant identified in this patient has been previously reported as a benign polymorphism (Rommel et al. 2002, 2005; Lerner-Ellis et al. 2014). Pathogenic variants in MYH11 cause familial thoracic aortic aneurysm (MIM \#132900) that is typically associated with aortic aneurysms but also strongly associated with patent ductus arteriosus not present in the patient or family members carrying the variant. Thus, the TGFBR2 VUS is the most likely candidate variant to be disease causal in our patient and we focused our analyses and experimentation to determine the clinical significance of this V419L variant.

\section{Structural Bioinformatics Analysis of the TGFBR2 V419L Variant}

The novel c.1255G>T variant in TGFBR2 is not observed in the Exome Sequencing (ESP), Exome Aggregation Consortium (ExAC), or Genome Aggregation Consortium (gnomAD) databases, and resides in the kinase domain of the protein (Fig. 3A). This valine residue is

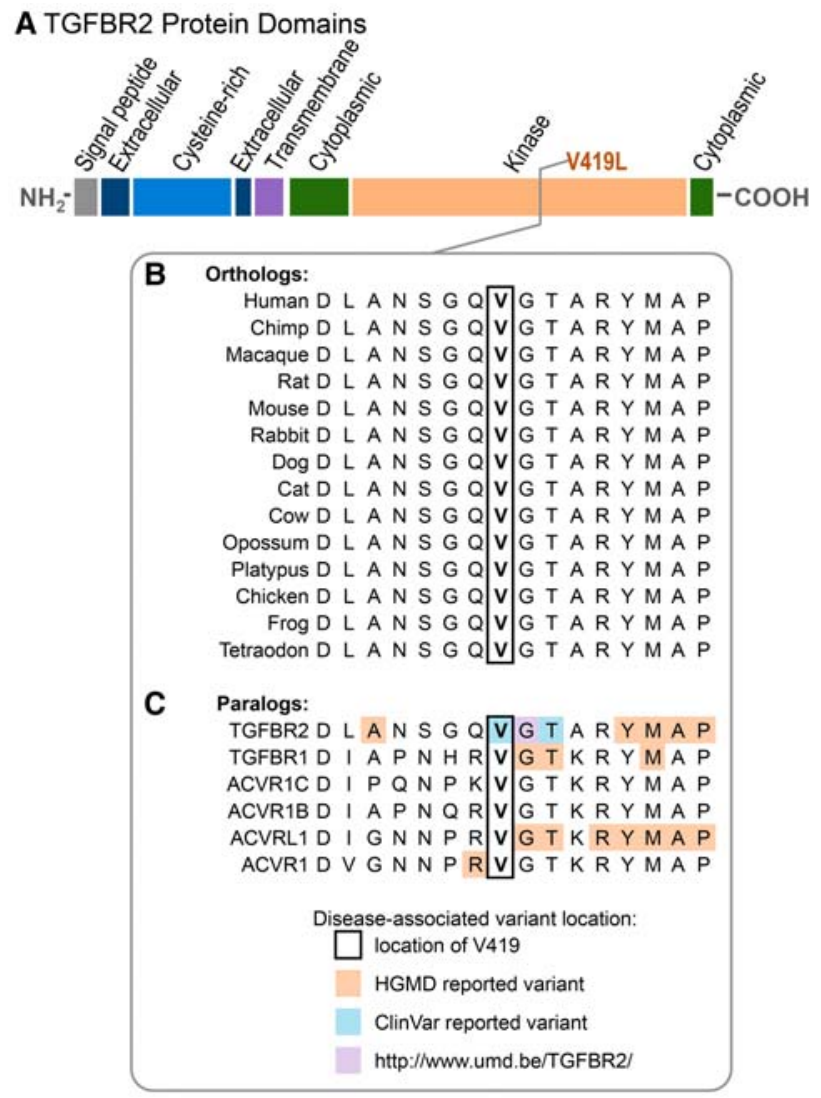

Figure 3. Protein domains and ortholog and paralog analyses. (A) V419 is located in the kinase domain and (B) is highly conserved in orthologs. (C) Paralog analysis reveals high conservation and disease-associated variants in nearby residues. V419E was reported as likely pathogenic in ClinVar. For a variant reported in more than one source, shading prioritization follows HGMD>ClinVar>UMD. Only variants reported as pathogenic or likely pathogenic in ClinVar were included. HGMD, Human Gene Mutation Database; UMD, Universal Mutation Database. 
highly conserved in vertebrates (Fig. 3B) as well as in human paralogs (Fig. 3C). Missense variants have been reported in nearby residues in TGFBR2 associated with disease by the Human Gene Mutation Database (HGMD) (Stenson et al. 2014), ClinVar (Landrum et al. 2016), and the Universal Mutation Database (Frederic et al. 2008) (http://www.umd.be/ TGFBR2/). We performed paralog analysis by mapping known pathogenic variants onto the multiple sequence alignment (MSA) (Fig. 3C) and show that pathogenic missense variants have also been reported in this region across paralogs. Additionally, there is only a single missense variant, L416V, in 1/120,972 alleles in ExAC (Lek et al. 2016) between residue positions 408 and 439 indicating a low rate of variation in this region. At the whole-gene level, TGFBR2 has fewer observed variants than expected $(z=2.25)$ but does not meet the criteria of the constraint metric from ExAC to be considered intolerant to missense variation (z-score $>3$ ). However, by interrogating only the region surrounding V419, we found the presence of disease-causal variants in TGFBR2 and its paralogs and a lack of variation in the ExAC data (assumed nondisease causal) for TGFBR2, suggesting that this is an important functional region of the gene/protein that may be intolerant to variation at a sub-gene level. Thus, variants in this region may be more likely to cause dysfunction and thereby disease, than elsewhere in the gene.

The in silico prediction tools (SIFT (Sorting Intolerant From Tolerant), PolyPhen [Polymorphism Phenotyping], and MutationTaster) are concordant in the result that this substitution has the potential to be deleterious to the protein. However, these tools are known to have a high false-positive rate for regions of high conservation (Kumar et al. 2012). Conversely, $\Delta \Delta G_{\text {fold }}$ calculation (Schymkowitz et al. 2005; Van Durme et al. 2011) yielded a value of $-0.11 \mathrm{kcal} / \mathrm{mol}$, which is an indication the substitution is unlikely to be destabilizing to the kinase domain. Consequently, we extended our studies to analyzing the potential effect of the V419L substitution using molecular modeling and molecular dynamics (MD) simulations, which have the ability to compare not only the structural changes but also the timedependent behavior of the variant in the tridimensional space and in comparison to the wild-type (WT) protein and other variants of known significance.

\section{Computational Biochemistry Methods Reveal Potential Alterations in the Structure} and Dynamics of the TGFBR2 V419L Variant

We pursued molecular modeling with MD simulations of the kinase domain of TGFBR2 using well-established molecular mechanic methodology (Fig. 4A). From our MD simulations, we quantified global changes in the structure using root-mean-square deviation (RMSD) and compared our patient's V419L to 30 previously reported pathogenic and likely pathogenic variants from ClinVar and HGMD (Fig. 4B). V419L exhibited a larger RMSD than WT and most pathogenic variants, indicating the conservative valine to leucine substitution may indeed have a large effect on the structure and dynamics of the protein. A principal component (PC) analysis of the MD simulations of TGFBR2 was completed to determine the differences in the major motions of the WT protein, the V419L VUSs, and the other known pathogenic variants (Fig. 4C,D). The first principal component (PC1) comprises $13.71 \%$ of the overall variance across all simulations and the second PC explains $9.69 \%$ (Fig. 4C). The motion encoded in PC1 for each residue is shown pictorially in Figure 4D. PC1 displays a motion wherein the N-lobe swivels or ratchets with respect to the C-lobe. In the +PC1 (-PC1) direction, the Gly-rich (Kornev et al. 2006) or P-loop (Foda et al. 2015) and the aC-helix move away from (toward) the activation loop, whereas components of the C-lobe shift in the opposite direction. By plotting representatives from our MD simulations in PC1 and PC2 coordinates, we show that expansion along PC1 is a strong component of the dynamic effects of V419L compared with WT; the space sampled during the WT and V419L MDs are essentially nonoverlapping (Fig. 4C). PC2 separates many of the reported pathogenic variants but contributes 
A
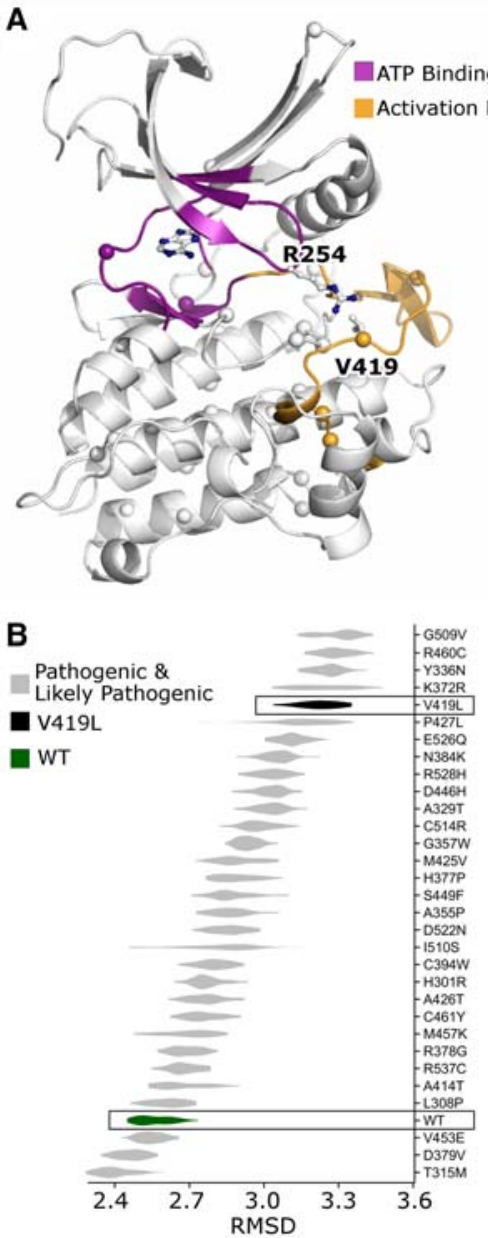

C

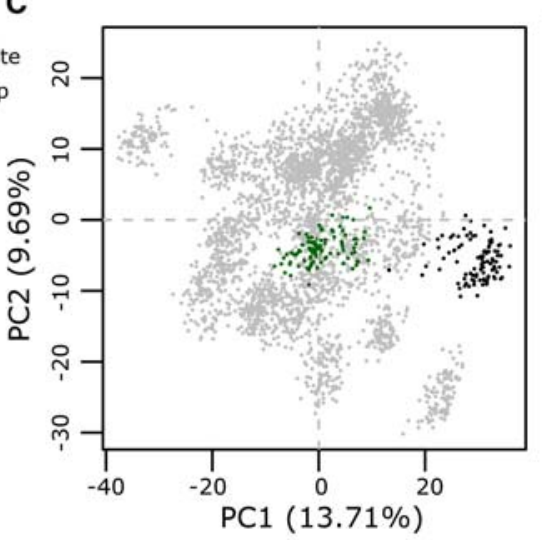

E

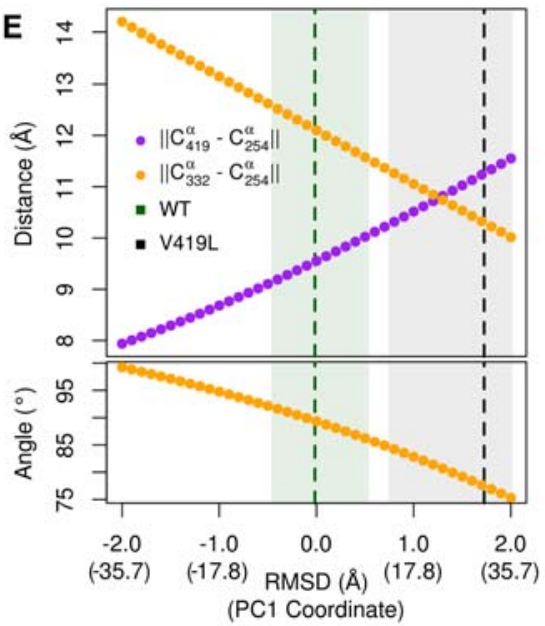

D

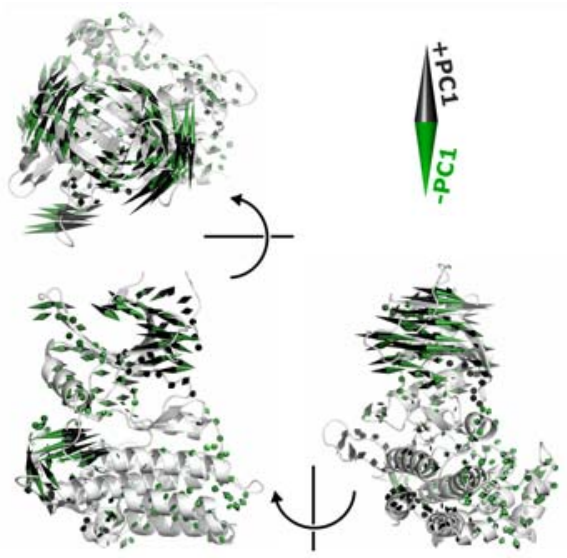

$\mathbf{F}$

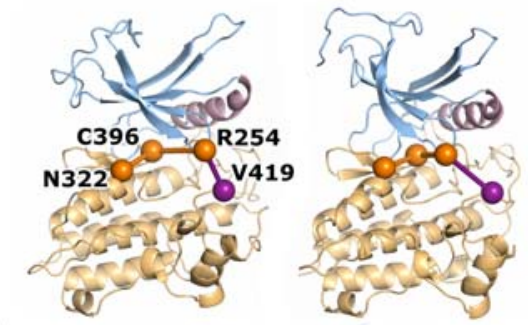

G

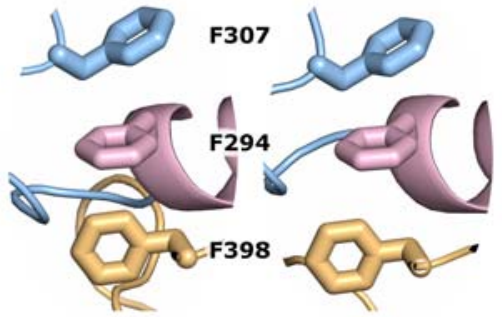

Figure 4. TGFBR2 structure and dynamics. (A) V419 is within the activation loop in close proximity to R254 in the ATP binding domain. Locations of pathogenic and likely pathogenic variants reported in ClinVar are marked by spheres. (B) Probability density of root-mean-square deviation (RMSD) from simulations demonstrated that V419L affects conformation. (C) Principal component analysis (PCA) reveals that V419L exhibits significant dynamic departures from wild type (WT) and many other pathogenic variants. Snapshots from each simulation are shown as points in the first two PCs. (D) The motion indicated by the first PC is shown, projected onto the initial WT structure. The direction and relative magnitude of each residue's motion is indicated-a twisting of the $\mathrm{N}$ - and C-lobes with respect to one another. V419L samples conformations that are further activated in the +PC1 direction than WT and most pathogenic variants. (E) We quantified the changes in reference distances along PC1. The median PC1 coordinates for WT and V419L simulations are indicated by dashed vertical lines and the full range of sampling by shaded regions. The angle of the ATP binding site is measured using N332-C396-R254. (F) We show the structure with the N-lobe colored blue, C-lobe orange, and $\alpha$ C-helix pink. Two conformations along PC1 are shown: a 2 A RMSD deformation in the negative direction (left) and a 2 A deformation in the positive direction (right). (G) Zooming in on the top of the hydrophobic spine reveals that PC1 also leads to misalignment of these residues. Thus, V419L may lead to enhanced sampling of an inactive state.

little to the difference between WT and V419L. Thus, PC analysis predicts that V419L leads to a dynamic shift in TGFBR2.

We also observe that during MD simulations, the correlation between motion along PC1, alteration of key reference distances, and movement of the aC-helix are visually evident. V419 comes into close proximity to the side chain of R254 that resides in the ATP binding 
domain on the end of the P-loop. For L419, the distance between the activation loop and the P-loop was increased (Fig. 4E,F). Thus, we quantified the change in proximity between the Ploop and the activation loop using a distance monitor and demonstrated that across the range of PC1 sampled by pathogenic variants, a difference of up to $4 \AA$ was observed, whereas for V419L these two structural features were an average of $2 \AA$ further separated than in WT (Fig. 4E). This difference is correlated with a closing of the ATP binding cavity by an average of $2 \AA$ and a change in the relative angle of the cavity opening by $15^{\circ}$. The PC1 motion also influences the distances between certain residues involved in the switched electrostatic network previously identified in other kinase structures (Ozkirimli et al. 2008). Further, the PC1 motion leads to alteration in the alignment of the hydrophobic spine and the orientation of the $\mathrm{aC}$-helix with respect to the activation loop (Fig. 4G). Both of these structural features have been previously and experimentally identified as hallmarks of the inactive state of other kinases (Kornev et al. 2006; Ozkirimli et al. 2008; Joseph et al. 2010). In our simulations, V419L leads to a propensity for TGFBR2 to sample conformations that exhibit features that bear resemblance to the canonical inactive conformation. Therefore, combining these results supports the prediction that V419L impairs the activity of TGFBR2, leading us to hypothesize the TGFBR2 signaling pathway will be disrupted and to perform biochemical assays to test the validity of this hypothesis.

\section{Cell-Based Functional Assays Demonstrate That the TGFBR2 V419L Variant Displays Aberrant Signaling Activity}

To test the hypothesis that the V419L variant disrupts the TGF- $\beta$ signaling pathway, we pursued in vitro cell-based studies measuring the downstream transcriptional activation using a luciferase reporter assay and the phosphorylation of SMAD2 (pSMAD2), which results from the activation of both cell-surface receptors in the canonical TGF- $\beta$ signaling pathway. We cotransfected a luciferase reporter construct containing a TGF- $\beta$ responsive element driving luciferase expression (p3TP-Lux) (Truty et al. 2009) with either WT or V419L TGFBR2 constructs in HCT116 cells that are deficient in endogenous TGFBR2 but contain TGFBR1 (Lee et al. 2013). Transfected cells with and without TGF- $\beta$ stimulation were measured with a luminometer for luciferase expression. Using this reporter assay, we show that the V419L variant-containing receptor has significantly reduced unstimulated and TGF- $\beta$-stimulated gene transcription associated with canonical TGF- $\beta$ signaling (Fig. 5A). Using western blot analyses to determine the ratio of PSMAD2 to the total amount of this protein, we show expression of the V419L-containing TGFBR2 is impaired in its ability to induce SMAD2 phosphorylation compared with WT upon stimulation with TGF- $\beta 1$ (Fig. 5B). These data are consistent with similarly evaluated disease-associated variants (Mizuguchi et al. 2004; Loeys et al. 2005; Horbelt et al. 2010) and confirm the hypothesis generated by bioinformatics, modeling, and MD simulations that the V419L substitution impairs the function of the protein and disrupts the TGF- $\beta$ signaling pathway.

\section{DISCUSSION}

We identified a patient with aortic root dilatation and bilateral iliac aneurysms carrying VUSs in three genes including TGFBR2, MYH11, and FBN1 by clinical gene-panel testing. The TGFBR2 variant was the most likely candidate for causing disease because of the vascular phenotype of the patient overlapping with LDS that is associated with heterozygous pathogenic variants in this gene, and, consequently, we carried out further studies to understand the impact of this variant on the protein and downstream signaling pathway.

The FBN1 variant was thought to be noncontributory because of previous reports of the variant being benign and its presence in unaffected controls. MYH11 is associated with aortic 
A TGFBR2 V419L has decreased TGF- $\beta$-related transcription

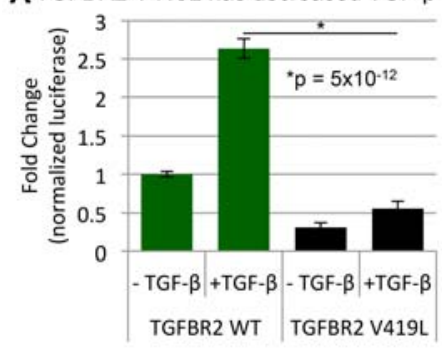

B TGFBR2 V419L has decreased SMAD2 phosphorylation

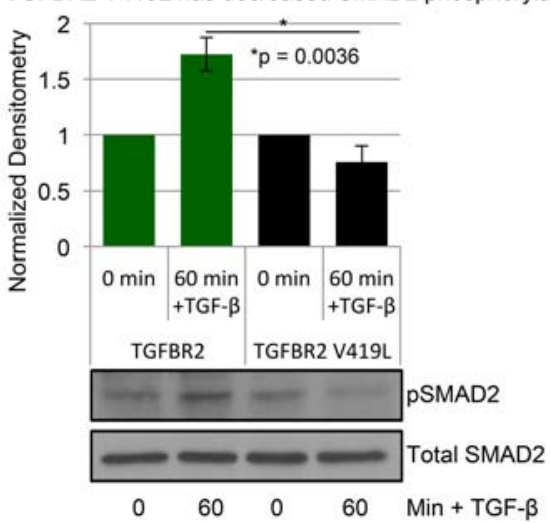

Figure 5. TGFBR2 V419L has decreased canonical TGF- $\beta$ signaling in vitro. Heterologous expression of TGFBR2 V419L in TGFBR2-deficient HCT116 cells leads to (A) decreased TGF- $\beta$-associated gene transcription using a luciferase reporter, P3TP-Lux, and (B) decreased phosphorylated SMAD2 following TGF- $\beta 1$ treatment compared with wild type. The bar graph represents the quantification of the western blot density. Each TGFBR2 WT and TGFBR2 mutant was normalized to its respective unstimulated $(0 \mathrm{~h})$ control.

aneurysms, and there is not enough evidence to exclude the possibility that it may be contributing to disease, but this family has no history of patent ductus arteriosus that is strongly associated with pathogenic variants in this gene making this variant a less likely candidate. VUSs in these genes cannot be interpreted as benign simply because an unaffected individual is determined to be a carrier of the variant because of the variable expressivity and reduced penetrance associated with the disease caused by these genes.

To determine the pathogenicity of the TGFBR2 V419L variant in our patient, we used a combination of variant annotation, in silico tools, and in vitro assays. V419 is located in the kinase domain where 91 of 99 missense variants reported in HGMD and 43 of the 44 likely pathogenic or pathogenic missense variants reported in ClinVar are located despite the fact that the kinase domain contains only $53 \%$ of the residues. We pursued ortholog and paralog comparisons and showed that the V419 residue is highly conserved across species and paralogs, indicating this residue might be important for protein function. By mapping previously reported pathogenic or likely pathogenic variants onto the paralog analysis, we were also able to show variation within this region of the TGFBR2 protein and its paralogs are often disease causal. V419L was not predicted to have a significant effect on the stability of the domain and, being a conservative hydrophobic alteration, is unlikely to directly impair biochemical properties of the activation loop. Atomic resolution protein modeling and MD simulation, however, suggest that $\mathrm{V} 419 \mathrm{~L}$ does have a large effect on function through alteration of structural features associated with experimentally verified inactive kinase conformations. Thus, even subtle changes in the amino acid sequence at certain positions in a protein may lead to dynamic shifts of the functional motions.

From the modeling and MD studies, we hypothesized the V419L variant will impact the function of the TGFBR2 protein despite the conservative amino acid substitution from valine to lysine (similar in size and biochemical properties). Previous studies have shown that disease-causing variants in TGFBR2 lead to loss of function and impairment of the canonical TGF- $\beta$ signaling pathway in heterologous systems (Mizuguchi et al. 2004; Loeys et al. 2005; Horbelt et al. 2010). The TGF- $\beta$ receptor complex is a tetramer of two TGFBR1 and two TGFBR2 proteins that binds the TGF- $\beta$ cytokine dimer leading to TGFBR1 phosphorylation and activation by the constitutively active TGFBR2. The activated TGFBR1 phosphorylates SMAD2, which dissociates from the receptor, interacts with SMAD4, translocates to the 
nucleus, and modulates gene transcription in the canonical TGF- $\beta$ signaling pathway (Fig. 1). We demonstrated that the V419L variant causes functional impairment of the TGFBR2 receptor by measuring the function of the canonical TGF- $\beta$ pathway. The patient's variant exhibited reduced TGF- $\beta$-associated transcriptional activation and reduced phosphorylation of SMAD2 after stimulation with TGF- $\beta$. The luciferase reporter construct, P3TP-Lux, used in this study has also been used previously to determine loss-of-function pathogenicity of other affected patient missense variants (Mizuguchi et al. 2004; Horbelt et al. 2010). Consistent with those findings, we showed that the V419L variant leads to decreased TGF- $\beta$-stimulated phosphorylation of SMAD2 that is a target of the activated TGFBR1/2 complex that associates with SMAD3 and SMAD4 to induce transcription in the nucleus. Decreased levels of pSMAD2 post-TGF- $\beta$ stimulation has previously been shown to result from pathogenic variants in TGFBR2 evaluated in a heterologous cell-based expression system (Horbelt et al. 2010). This confirms the hypothesis that the valine to leucine substitution at position 419 causes a loss of function to TGFBR2 and disrupts the downstream signaling cascade.

Interestingly, in patient tissue samples, TGF- $\beta$-related heritable connective tissue disorders are associated with an increase in TGF- $\beta$ signaling. TGF- $\beta$ up-regulation is expected for variants causing loss of function in FBN1 and SKI. Deficiency of FBN1 leads to a reduced ability to properly sequester TGF- $\beta$ ligand in the extracellular matrix and the predicted increase in TGF- $\beta$ signaling caused by the overabundance of the free ligand (Neptune et al. 2003; Franken et al. 2013) has been shown to be a key part of the disease mechanism ( $\mathrm{Ng}$ et al. 2004; Habashi et al. 2006; Cohn et al. 2007). Decreases in function of the TGF- $\beta$-signaling repressor, SKI, also lead to an expected increase in TGF- $\beta$ signaling because of its reduced ability to prevent the PSMAD2/3-SMAD4 from translocating to the nucleus to activate transcription (Doyle et al. 2012). The remaining genes known to cause disease by dysregulating TGF- $\beta$ signaling, including TGFBR1, TGFBR2, SMAD3, TGFB2, TGFB3, and SMAD2, are thought to be pathogenic by a loss of function of the encoded protein, but the evaluation of patient tissue identified a paradoxical up-regulation of the TGF- $\beta$ signaling pathway (Loeys et al. 2005; van de Laar et al. 2011; Boileau et al. 2012; Doyle et al. 2012; Lindsay et al. 2012; Bertoli-Avella et al. 2015; Micha et al. 2015). The mechanism of this unexpected up-regulation warrants further investigation but is thought to be related to dysregulation of an inhibitory feedback mechanism and increased signaling through a noncanonical pathway (Lindsay and Dietz 2011). We expect the patient we describe in this study to have TGF- $\beta$ upregulation consistent with these previous findings, but tissue was unavailable for evaluation.

In this study, we have combined extensive annotation with in silico, and in vitro techniques to functionally characterize a candidate VUS. The V419L variant in TGFBR2 was shown to impact the protein function by inducing changes to protein structure and dynamics, leading to decreased TGF- $\beta$ signaling. These findings are consistent with previously identified pathogenic variants in TGFBR2. The ACMG 2015 guidelines (Richards et al. 2015) were used to assess the pathogenicity of the V419L variant and determined it should be classified as likely pathogenic (Table 2) providing a genetic diagnosis of LDS for our patient. This diagnosis enables proper long-term management of our patient, including blood pressure control, vascular imaging surveillance, and surgical interventions, based on published guidelines (MacCarrick et al. 2014). It further allows for the testing and counseling of at risk family members.

\section{METHODS}

\section{Genetic Testing}

To determine the genetic etiology of disease in the patient, clinical gene-panel testing was pursued through GeneDx. Methods for the Thoracic Aortic Aneurysm and Dissection 
Table 2. TGFBR2 V419L is likely pathogenic by 2015 ACMG guidelines

\begin{tabular}{|c|c|c|}
\hline Category & Description & Evidence \\
\hline PS3 & $\begin{array}{l}\text { Well-established in vitro or in vivo functional } \\
\text { studies supportive of a damaging effect on } \\
\text { the gene or gene product }\end{array}$ & $\begin{array}{l}\text { Cells expressing the variant have decreased } \\
\text { TGF- } \beta \text {-related transcription and decreased } \\
\text { SMAD2 phosphorylation compared with } \\
\text { wild type. }\end{array}$ \\
\hline PM2 & $\begin{array}{l}\text { Absent from controls (or at extremely low } \\
\text { frequency if recessive) in ESP, 1000G, or } \\
\text { ExAC }\end{array}$ & $\begin{array}{l}\text { The variant is absent in the publicly available } \\
\text { databases. }\end{array}$ \\
\hline PP2 & $\begin{array}{l}\text { Missense variant in a gene that has a low rate } \\
\text { of benign missense variation and in which } \\
\text { missense variants are a common } \\
\text { mechanism of disease }\end{array}$ & $\begin{array}{l}\text { Missense variants in TGFBR2 have been } \\
\text { shown to cause disease. }\end{array}$ \\
\hline PP3 & $\begin{array}{l}\text { Multiple lines of computational evidence } \\
\text { support a deleterious effect on the gene or } \\
\text { gene product (conservation, evolutionary, } \\
\text { splicing impact, etc.) }\end{array}$ & $\begin{array}{l}\text { In silico prediction algorithms as well as in } \\
\text { silico protein modeling support a } \\
\text { deleterious effect of this variant. }\end{array}$ \\
\hline
\end{tabular}

ACMG, American College of Medical Genetics and Genomics; ESP, Exome Sequencing Project; 1000G, 1000 Genomes; ExAC, Exome Aggregation Consortium.

(TAAD)/Marfan syndrome/Related Disorders Sequencing and Deletion/Duplication Panel clinical test are available at https://www.genedx.com/wp-content/uploads/crm_docs/Info_ sheet_Marfan_TAAD.pdf.

\section{Paralog Analysis}

The paralog annotation method was used to provide insight into the potential association of the V419L variant with previously described deleterious changes in TGFBR2 and paralogous proteins. This method uses MSA of paralogs for gene ENSG00000163513 (transcript: ENST00000295754; GRCh37.p13) as defined by Ensembl (Cunningham et al. 2015). The MSA was constructed using Clustal Omega (Sievers et al. 2011; McWilliam et al. 2013) with default parameters. Published variants in paralogs were obtained from ClinVar (Landrum et al. 2014, 2016), HGMD Professional version 2016.3 (Stenson et al. 2014), and the Universal Mutation Database (Frederic et al. 2008) (http://www.umd.be/TGFBR2/).

\section{Molecular Modeling}

Because no experimental structure of TGFBR2 exists, we queried to the PDB (Berman et al. 2000) using T-Coffee (Di Tommaso et al. 2011) and generated a homology-based model against the best available template using MODELLER (Sali and Blundell 1993) version 9.15. The template of adenine-bound ACVR2B, 2OLU (Han et al. 2007) exhibited $46 \%$ sequence identity for the modeled region. We generated 20 models and selected the model with the lowest DOPE (discrete optimized protein energy) score (Shen and Sali 2006) for further analyses. Model quality was assessed using PROCHECK (Laskowski et al. 1996), QMEAN (Benkert et al. 2009) and VADAR version 1.8 (Willard et al. 2003). Pathogenic and likely pathogenic missense variants were extracted from ClinVar (Landrum et al. 2014) and mapped to our TGFBR2 model. In silico mutagenesis was performed using Discovery Studio (Dassault Systèmes BIOVIA, Discovery Studio Modeling Environment, Release 2017, San Diego: Dassault Systèmes, 2017). Changes in folding energy upon mutation $\left(\Delta \Delta G_{\text {fold }}\right)$ were computed using FoldX version 4 (Schymkowitz et al. 2005; Van Durme et al. 2011). 


\section{Simulations}

The CHARMM c36b2 all-atom force field (Cornell et al. 1995) was used for TGFBR2 MD simulations. The environment was modeled using a distance-dependent implicit solvent model, Generalized Bourne with Simple Switching (Feig et al. 2004; Chocholousova and Feig 2006) as implemented in Discovery Studio, with a dielectric constant of 80 and a pH of 7.4. Each model was energy minimized for 5000 steps of the steepest descent followed by adaptive conjugate gradient, enforcing a maximum RMSD for each procedure of 1.0 and 0.2 , respectively, using the SHAKE (Ryckaert et al. 1977) procedure. The minimized TGFBR2 kinase models were heated to $300^{\circ} \mathrm{K}$ over 300 psec using a 2-fsec time step and equilibrated for $2 \mathrm{nsec}$. A further $10 \mathrm{nsec}$ of simulation was generated, with conformations saved every 20 psec. Trajectories were aligned to the initial WT conformation prior to analysis. RMSD and root mean-square fluctuation values were calculated at the residue level using $\mathrm{C}^{\alpha}$ atoms. PC analysis was performed in Cartesian space using $C^{\alpha}$ atoms. Analyses were carried out in the R programming language ( $R$ Core Team 2014), leveraging the Bio3d (Grant et al. 2006) package version 2.2.4. Molecular visualizations were generated using PyMOL (The PyMOL Molecular Graphics System, Version 1.5.0.3, Schrödinger, LLC.).

\section{Reporter Assays}

Luciferase-based reporter assays were performed using the HCT116 human colon carcinoma cell line, which was maintained in RPMI (Roswell Park Memorial Institute) medium with 10\% fetal bovine serum and antibiotics. Cells were grown at $37^{\circ} \mathrm{C}$ with $5 \% \mathrm{CO}_{2}$ in a humidified incubator. The TGFBR2 expression plasmid (NM_003242.5) (Truty et al. 2009) was mutated at valine 419 to leucine (hereafter denoted TGFBR2 V419L) with the Quik Change XL II site directed mutagenesis kit (Agilent). The activity of the WT TGFBR2 and mutant TGFBR2 V419L receptors was monitored via luciferase reporter assay. HCT116 cells at $5 \times 10^{6}$ cells $/ \mathrm{ml}$ in serum free media were mixed with $3 \mu \mathrm{g}$ of reporter construct (p3TP-Lux reporter plasmid containing TGF- $\beta$ responsive elements) (Truty et al. 2009) and $9 \mu \mathrm{g}$ of each WT and mutant TGFBR2. Cells were transfected by electroporation on a BTX Model 820 square wave electroporator at $250 \mathrm{~V}$, two pulses for $13 \mathrm{msec}$ with a 1-sec interval and plated with serum in triplicate on a six-well dish. After cells expressed constructs for $24 \mathrm{~h}$, media was changed to no serum and the receptor was stimulated with TGF- $\beta 1$ (5 ng/ml; R\&D Systems 240-B-002) for an additional $24 \mathrm{~h}$. Protein lysates were isolated at $48 \mathrm{~h}$ postelectroporation and relative luciferase expression assayed on a Berthold Lumat LB 9507 luminometer using Promega's Dual Luciferase Reporter Assay System. Total protein concentration was used to control luciferase activity and all conditions were normalized to unstimulated cells overexpressing WT TGFBR2. Four independent experiments were completed with t-tests indicating significance between conditions.

\section{SMAD2 Phosphorylation Assay}

HCT116 cells $\left(4 \times 10^{6}\right)$ were electroporated with TGFBR2 constructs as described for the luciferase assay and plated with serum onto 60-mm dishes. After $24 \mathrm{~h}$, cells were serum starved for 12-14 $\mathrm{h}$ and then stimulated with TGF- $\beta 1(5 \mathrm{ng} / \mathrm{ml})$ to initiate the signaling cascade. Cells were lysed in RIPA-150 buffer with protease and phosphatase inhibitors (Thermo Fisher Scientific) after $60 \mathrm{~min}$ of stimulation. Equal protein $(20 \mu \mathrm{g})$ was loaded for each sample and separated by $12 \%$ sodium dodecyl sulfate-polyacrylamide gel electrophoresis (SDSPAGE), transferred to nitrocellulose membrane. Western blots were blocked with $3 \%$ bovine serum albumin (BSA) and probed overnight with primary antibodies to total SMAD2 (1:1000, Cell Signaling 5339S), pSMAD2 (1:500, Cell Signaling 3101S), and $\gamma$-tubulin (confirmed equal loading, Supplemental Figure 1; 1:1000; Sigma-Aldrich T3559) and developed with chemiluminescence (Papadakis et al. 2015). ImageJ was used to quantify western blot 
Competing Interest Statement The authors have declared no competing interest.

\section{Referees}

William A. Gahl

Anonymous

Received January 6, 2017; accepted in revised form April 12, 2017. density and each TGFBR2 WT and TGFBR2 mutant was normalized to its respective unstimulated $(\mathrm{O}$ h) control. Four independent experiments were completed with $t$-tests indicating significance between conditions.

\section{ADDITIONAL INFORMATION}

\section{Data Deposition and Access}

The variants identified in this patient were submitted to ClinVar (http://www.ncbi.nlm.nih. gov/clinvar/) by the testing institution (GeneDx) under accession numbers SCV0002509 40.9, SCV000233776.7, and SCV000234882.8. Sequence data were unable to be submitted to publicly available databases because these data were collected by the clinical reference testing institution without patient consent to do so.

\section{Ethics Statement}

The patient provided written informed consent to research protocol \# 12-009346 approved by the Mayo Clinic Institutional Review Board for this study and publication of this paper.

\section{Acknowledgments}

We thank the patient for participation in this study.

\section{Author Contributions}

M.A.C., R.A.U., D.R.D., and E.W.K. conceived of and designed the study. M.T.Z., G.R.O., and R.A.U. generated and analyzed the protein modeling and molecular dynamic simulation data. M.A.C., E.W.K., M.T.Z., and R.A.U. interpreted these findings. A.J.M., G.A.L., and R.A.U. generated and analyzed the in vitro data. M.A.C., E.W.K., A.J.M., G.A.L., and R.A.U. interpreted the cell-based findings. D.R.D. clinically evaluated and counseled the patient, obtained consent from the patient, and collected the clinical and imaging data. P.R.B., N.J.B., and G.R.O. contributed to the study design and data interpretation. M.A.C. and M.T.Z. drafted the manuscript, and all authors critically reviewed the manuscript.

\section{Funding}

This work was generously supported by the Center for Individualized Medicine at Mayo Clinic.

\section{REFERENCES}

Benkert P, Kunzli M, Schwede T. 2009. QMEAN server for protein model quality estimation. Nucleic Acids Res 37: W510-W514.

Berman HM, Westbrook J, Feng Z, Gilliland G, Bhat TN, Weissig H, Shindyalov IN, Bourne PE. 2000. The Protein Data Bank. Nucleic Acids Res 28: 235-242.

Bertoli-Avella AM, Gillis E, Morisaki H, Verhagen JM, de Graaf BM, van de Beek G, Gallo E, Kruithof BP, Venselaar H, Myers LA, et al. 2015. Mutations in a TGF- $\beta$ ligand, TGFB3, cause syndromic aortic aneurysms and dissections. J Am Coll Cardiol 65: 1324-1336.

Boileau C, Guo DC, Hanna N, Regalado ES, Detaint D, Gong L, Varret M, Prakash SK, Li AH, d'Indy H, et al. 2012. TGFB2 mutations cause familial thoracic aortic aneurysms and dissections associated with mild systemic features of Marfan syndrome. Nat Genet 44: 916-921.

Chocholousova J, Feig M. 2006. Balancing an accurate representation of the molecular surface in generalized born formalisms with integrator stability in molecular dynamics simulations. J Comput Chem 27: 719-729. 
Cohn RD, van Erp C, Habashi JP, Soleimani AA, Klein EC, Lisi MT, Gamradt M, Ap Rhys CM, Holm TM, Loeys BL, et al. 2007. Angiotensin II type 1 receptor blockade attenuates TGF- $\beta$-induced failure of muscle regeneration in multiple myopathic states. Nat Med 13: 204-210.

Cornell WD, Cieplak P, Bayly Cl, Gould IR, Merz KM, Ferguson DM, Spellmeyer DC, Fox T, Caldwell JW, Kollman PA. 1995. A second generation force field for the simulation of proteins, nucleic acids, and organic molecules. J Am Chem Soc 117: 5179-5197.

Cunningham F, Amode MR, Barrell D, Beal K, Billis K, Brent S, Carvalho-Silva D, Clapham P, Coates G, Fitzgerald S, et al. 2015. Ensembl 2015. Nucleic Acids Res 43: D662-D669.

De Backer J, Renard M, Campens L, Francois K, Callewaert B, Coucke P, De Paepe A. 2013. Genes in thoracic aortic aneurysms and dissections-do they matter?: translation and integration of research and modern genetic techniques into daily clinical practice. Aorta (Stamford) 1: 135-145.

Di Tommaso P, Moretti S, Xenarios I, Orobitg M, Montanyola A, Chang JM, Taly JF, Notredame C. 2011. TCoffee: a web server for the multiple sequence alignment of protein and RNA sequences using structural information and homology extension. Nucleic Acids Res 39: W13-W17.

Doyle AJ, Doyle JJ, Bessling SL, Maragh S, Lindsay ME, Schepers D, Gillis E, Mortier G, Homfray T, Sauls K, et al. 2012. Mutations in the TGF- $\beta$ repressor SKI cause Shprintzen-Goldberg syndrome with aortic aneurysm. Nat Genet 44: 1249-1254.

Feig M, Onufriev A, Lee MS, Im W, Case DA, Brooks CL III. 2004. Performance comparison of generalized born and Poisson methods in the calculation of electrostatic solvation energies for protein structures. $J$ Comput Chem 25: 265-284.

Foda ZH, Shan Y, Kim ET, Shaw DE, Seeliger MA. 2015. A dynamically coupled allosteric network underlies binding cooperativity in Src kinase. Nat Commun 6: 5939.

Franken R, den Hartog AW, de Waard V, Engele L, Radonic T, Lutter R, Timmermans J, Scholte AJ, van den Berg MP, Zwinderman AH, et al. 2013. Circulating transforming growth factor- $\beta$ as a prognostic biomarker in Marfan syndrome. Int J Cardiol 168: 2441-2446.

Frederic MY, Hamroun D, Faivre L, Boileau C, Jondeau G, Claustres M, Beroud C, Collod-Beroud G. 2008. A new locus-specific database (LSDB) for mutations in the TGFBR2 gene: UMD-TGFBR2. Hum Mutat 29: 33-38.

Global Genes. 2016. RARE diseases: facts and statistics. Retrieved 12/12/2016, from https:// globalgenes.org/ rare-diseases-facts-statistics/.

Grant BJ, Rodrigues AP, ElSawy KM, McCammon JA, Caves LS. 2006. Bio3d: an R package for the comparative analysis of protein structures. Bioinformatics 22: 2695-2696.

Habashi JP, Judge DP, Holm TM, Cohn RD, Loeys BL, Cooper TK, Myers L, Klein EC, Liu G, Calvi C, et al. 2006. Losartan, an AT1 antagonist, prevents aortic aneurysm in a mouse model of Marfan syndrome. Science 312: 117-121.

Han S, Loulakis P, Griffor M, Xie Z. 2007. Crystal structure of activin receptor type IIB kinase domain from human at 2.0 Angstrom resolution. Protein Sci 16: 2272-2277.

Horbelt D, Guo G, Robinson PN, Knaus P. 2010. Quantitative analysis of TGFBR2 mutations in Marfan-syndrome-related disorders suggests a correlation between phenotypic severity and Smad signaling activity. J Cell Sci 123: 4340-4350.

Joseph RE, Xie Q, Andreotti AH. 2010. Identification of an allosteric signaling network within Tec family kinases. J Mol Biol 403: 231-242.

Kornev AP, Haste NM, Taylor SS, Eyck LF. 2006. Surface comparison of active and inactive protein kinases identifies a conserved activation mechanism. Proc Natl Acad Sci 103: 17783-17788.

Kumar S, Sanderford M, Gray VE, Ye J, Liu L. 2012. Evolutionary diagnosis method for variants in personal exomes. Nat Methods 9: 855-856.

Landrum MJ, Lee JM, Riley GR, Jang W, Rubinstein WS, Church DM, Maglott DR. 2014. ClinVar: public archive of relationships among sequence variation and human phenotype. Nucleic Acids Res 42: D980-D985.

Landrum MJ, Lee JM, Benson M, Brown G, Chao C, Chitipiralla S, Gu B, Hart J, Hoffman D, Hoover J, et al. 2016. ClinVar: public archive of interpretations of clinically relevant variants. Nucleic Acids Res 44: D862-D868.

Laskowski RA, Rullmannn JA, MacArthur MW, Kaptein R, Thornton JM. 1996. AQUA and PROCHECKNMR: programs for checking the quality of protein structures solved by NMR. J Biomol NMR 8: 477-486.

Lee J, Ballikaya S, Schonig K, Ball CR, Glimm H, Kopitz J, Gebert J. 2013. Transforming growth factor $\beta$ receptor 2 (TGFBR2) changes sialylation in the microsatellite unstable (MSI) Colorectal cancer cell line HCT116. PLoS One 8: e57074.

Lek M, Karczewski KJ, Minikel EV, Samocha KE, Banks E, Fennell T, O’Donnell-Luria AH, Ware JS, Hill AJ, Cummings BB, et al. 2016. Analysis of protein-coding genetic variation in 60,706 humans. Nature 536: 285-291. 
Lerner-Ellis JP, Aldubayan SH, Hernandez AL, Kelly MA, Stuenkel AJ, Walsh J, Joshi VA. 2014. The spectrum of FBN1, TGF $\beta R 1, T G F \beta R 2$ and ACTA2 variants in 594 individuals with suspected Marfan syndrome, LoeysDietz syndrome or thoracic aortic aneurysms and dissections (TAAD). Mol Genet Metab 112: 171-176.

Lindsay ME, Dietz HC. 2011. Lessons on the pathogenesis of aneurysm from heritable conditions. Nature 473: 308-316.

Lindsay ME, Schepers D, Bolar NA, Doyle JJ, Gallo E, Fert-Bober J, Kempers MJ, Fishman EK, Chen Y, Myers L, et al. 2012. Loss-of-function mutations in TGFB2 cause a syndromic presentation of thoracic aortic aneurysm. Nat Genet 44: 922-927.

Loeys BL, Chen J, Neptune ER, Judge DP, Podowski M, Holm T, Meyers J, Leitch CC, Katsanis N, Sharifi N, et al. 2005. A syndrome of altered cardiovascular, craniofacial, neurocognitive and skeletal development caused by mutations in TGFBR1 or TGFBR2. Nat Genet 37: 275-281.

Loeys BL, Dietz HC, Braverman AC, Callewaert BL, De Backer J, Devereux RB, Hilhorst-Hofstee Y, Jondeau G, Faivre L, Milewicz DM, et al. 2010. The revised Ghent nosology for the Marfan syndrome. J Med Genet 47: 476-485.

MacCarrick G, Black JH III, Bowdin S, El-Hamamsy I, Frischmeyer-Guerrerio PA, Guerrerio AL, Sponseller PD, Loeys B, Dietz HC III. 2014. Loeys-Dietz syndrome: a primer for diagnosis and management. Genet Med 16: 576-587.

McWilliam H, Li W, Uludag M, Squizzato S, Park YM, Buso N, Cowley AP, Lopez R. 2013. Analysis Tool Web Services from the EMBL-EBI. Nucleic Acids Res 41: W597-W600.

Micha D, Guo DC, Hilhorst-Hofstee Y, van Kooten F, Atmaja D, Overwater E, Cayami FK, Regalado ES, van Uffelen R, Venselaar $\mathrm{H}$, et al. 2015. SMAD2 mutations are associated with arterial aneurysms and dissections. Hum Mutat 36: 1145-1149.

Mizuguchi T, Collod-Beroud G, Akiyama T, Abifadel M, Harada N, Morisaki T, Allard D, Varret M, Claustres M, Morisaki $\mathrm{H}$, et al. 2004. Heterozygous TGFBR2 mutations in Marfan syndrome. Nat Genet 36: 855-860.

Neptune ER, Frischmeyer PA, Arking DE, Myers L, Bunton TE, Gayraud B, Ramirez F, Sakai LY, Dietz HC. 2003. Dysregulation of TGF- $\beta$ activation contributes to pathogenesis in Marfan syndrome. Nat Genet 33: 407-411.

Ng CM, Cheng A, Myers LA, Martinez-Murillo F, Jie C, Bedja D, Gabrielson KL, Hausladen JM, Mecham RP, Judge DP, et al. 2004. TGF- $\beta$-dependent pathogenesis of mitral valve prolapse in a mouse model of Marfan syndrome. J Clin Invest 114: 1586-1592.

Ozkirimli E, Yadav SS, Miller WT, Post CB. 2008. An electrostatic network and long-range regulation of Src kinases. Protein Sci 17: 1871-1880.

Papadakis KA, Krempski J, Reiter J, Svingen P, Xiong Y, Sarmento OF, Huseby A, Johnson AJ, Lomberk GA, Urrutia RA, et al. 2015. Kruppel-like factor KLF10 regulates transforming growth factor receptor II expression and TGF- $\beta$ signaling in CD8 ${ }^{+} \mathrm{T}$ lymphocytes. Am J Physiol Cell Physiol 308: C362-C371.

R Core Team. 2014. R: a language and environment for statistical computing. R Foundation for Statistical Computing. http://www.R-project.org.

Richards S, Aziz N, Bale S, Bick D, Das S, Gastier-Foster J, Grody WW, Hegde M, Lyon E, Spector E, et al. 2015. Standards and guidelines for the interpretation of sequence variants: a joint consensus recommendation of the American College of Medical Genetics and Genomics and the Association for Molecular Pathology. Genet Med 17: 405-424.

Rommel K, Karck M, Haverich A, Schmidtke J, Arslan-Kirchner M. 2002. Mutation screening of the fibrillin-1 (FBN1) gene in 76 unrelated patients with Marfan syndrome or Marfanoid features leads to the identification of 11 novel and three previously reported mutations. Hum Mutat 20: 406-407.

Rommel K, Karck M, Haverich A, von Kodolitsch Y, Rybczynski M, Muller G, Singh KK, Schmidtke J, ArslanKirchner M. 2005. Identification of 29 novel and nine recurrent fibrillin-1 (FBN1) mutations and genotype-phenotype correlations in 76 patients with Marfan syndrome. Hum Mutat 26: 529-539.

Ryckaert JP, Ciccotti G, Berendsen HJC. 1977. Numerical integration of the Cartesian equations of motion of a system with constraints: molecular dynamics of n-alkanes. J Comput Phys 23: 327-341.

Sali A, Blundell T. 1993. Comparative protein modelling by satisfaction of spatial restraints. J Mol Biol 234: 779-815.

Schymkowitz J, Borg J, Stricher F, Nys R, Rousseau F, Serrano L. 2005. The FoldX web server: an online force field. Nucleic Acids Res 33: W382-W388.

Shen MY, Sali A. 2006. Statistical potential for assessment and prediction of protein structures. Protein Sci 15: 2507-2524.

Sievers F, Wilm A, Dineen D, Gibson TJ, Karplus K, Li W, Lopez R, McWilliam H, Remmert M, Soding J, et al. 2011. Fast, scalable generation of high-quality protein multiple sequence alignments using Clustal $\omega$. Mol Syst Biol 7: 539.

Stenson PD, Mort M, Ball EV, Shaw K, Phillips A, Cooper DN. 2014. The Human Gene Mutation Database: building a comprehensive mutation repository for clinical and molecular genetics, diagnostic testing and personalized genomic medicine. Hum Genet 133: 1-9. 
Truty MJ, Lomberk G, Fernandez-Zapico ME, Urrutia R. 2009. Silencing of the transforming growth factor- $\beta$ (TGF $\beta$ ) receptor II by Kruppel-like factor 14 underscores the importance of a negative feedback mechanism in TGF $\beta$ signaling. J Biol Chem 284: 6291-6300.

van de Laar IM, Oldenburg RA, Pals G, Roos-Hesselink JW, de Graaf BM, Verhagen JM, Hoedemaekers YM, Willemsen R, Severijnen LA, Venselaar H, et al. 2011. Mutations in SMAD3 cause a syndromic form of aortic aneurysms and dissections with early-onset osteoarthritis. Nat Genet 43: 121-126.

Van Durme J, Delgado J, Stricher F, Serrano L, Schymkowitz J, Rousseau F. 2011. A graphical interface for the FoldX forcefield. Bioinformatics 27: 1711-1712.

Verstraeten A, Alaerts M, Van Laer L, Loeys B. 2016. Marfan syndrome and related disorders: 25 years of gene discovery. Hum Mutat 37: 524-531.

Willard L, Ranjan A, Zhang H, Monzavi H, Boyko R, Sykes B, Wishart D. 2003. VADAR: a web server for quantitative evaluation of protein structure quality. Nucleic Acids Res 31: 3316-3319. 


\section{COLD SPRING HARBOR Molecular Case Studies}

\section{Functional validation reveals the novel missense V419L variant in TGFBR2 associated with Loeys-Dietz syndrome (LDS) impairs canonical TGF- $\beta$ signaling}

Margot A. Cousin, Michael T. Zimmermann, Angela J. Mathison, et al.

Cold Spring Harb Mol Case Stud 2017, 3: a001727 originally published online April 14, 2017

Access the most recent version at doi: $10.1101 / \mathrm{mcs} . a 001727$

Supplementary http://molecularcasestudies.cshlp.org/content/suppl/2017/05/09/mcs.a001727.D
Material

References This article cites 54 articles, 6 of which can be accessed free at: http://molecularcasestudies.cshlp.org/content/3/4/a001727.full.html\#ref-list-1

License This article is distributed under the terms of the Creative Commons Attribution-NonCommercial License, which permits reuse and redistribution, except for commercial purposes, provided that the original author and source are credited.

Email Alerting Receive free email alerts when new articles cite this article - sign up in the box at the Service top right corner of the article or click here. 